\title{
Psychological impact of pre-operative education on post- operative health in patients undergoing elective hysterectomy
}

\author{
Shuchi Jain, Shruti Pansare \\ Corresponding author: Dr. Shuchi Jain, A/13 Dhanwantri Nagar, Sewagram, Wardha, \\ Maharashtra, India; Email : shuchimjain@gmail.com
}

Distributed under Attribution-Non Commercial - Share Alike 4.0 International (CC BY-NC-SA 4.0)

\begin{abstract}
Objectives: The objective of the study was to assess the impact of pre-operative education on post-operative psychosocial health. Methods: This study was conducted in a medical teaching hospital in rural area of central India. It was an interventional research in 96 women reporting to the gynecology ward of department of obstetrics and gynecology of a teaching hospital for hysterectomy operation. They were randomly divided into two groups; wherein the patients of one group are imparted with the knowledge of the surgery, its types, surgical procedure, duration, preoperative preparation, post-operative recovery and effects of the surgery and the other given routine preoperative advice. With the help of HADS (hospital anxiety depression scale), pre-operative as well as post-operative anxiety and depression was assessed. Result: The mean score of intervention group for pre-operative anxiety was $6.81 \pm 2.69$ as compared to $9.13 \pm 3.36$ in the control group while post-operative scores were $6.79 \pm 3.46$ and $9.71 \pm 3.16$ respectively. In case of depression, there was rise in score in control group from $9.93 \pm 4.55$ pre-operatively to $10.48 \pm 4.82$ post-operatively. In intervention group it fell from $8.02 \pm 3.46$ pre-operatively to $6.85 \pm 3.98$ postoperatively. Conclusion: These results signify that there is a definite relationship between pre-operative education and postoperative early recovery as the anxiety is lowered and patients are well aware about the consequences of the surgery and are psychological fit to accept the results of the surgery.
\end{abstract}

Keywords: Anxiety, depression, HADS scale, post-operative recovery.

Women who undergo hysterectomy face a multitude of physical and psychosocial problems both before and after the hysterectomy. There are various aspects like anxiety; both pre and post-operative, familiar support, social misconceptions, psychological aspects. While it is established that side effects of hysterectomy include both positive and negative physical or emotional aspects, there have been no empirical studies examining these effects among Indian rural women. No psychological preparation for the operation, absence of closest people in the decision making before the operation, lack of knowledge on the surgical operational span, lacking psychological aid after the operation can significantly affect the rehabilitation and the process of regaining the social functions. Few studies have shown that preoperative integrated intervention based on psychological health education can improve peri-operative negative emotions and psychological stress in young women undergoing hysterectomy ${ }^{1}$. In few cases preoperative assessment and counseling is a key to optimizing postoperative psychological and sexual outcomes ${ }^{2}$. Few studies shows presurgical psychopathology is a predictive of postsurgical psychopathology ${ }^{3}$.

Patients may perceive the day of surgery as the biggest and the most threatening day in their lives. The degree to which each patient manifest anxiety related to in hospital experiences depends on many factors. These include age, gender, type and extent of the proposed surgery, previous surgical experience, and personal susceptibility to stressful situations. Some degree of anxiety is a natural reaction to the unpredictable and potentially threatening circumstances

Received: $24^{\text {th }}$ July 2020, Peer review completed: $21^{\text {th }}$ September 2020, Accepted: $\mathbf{1}^{\text {st }}$ October 2020.

Jain S, Pansare S. Psychological impact of pre-operative education on post-operative health in patients undergoing elective hysterectomy. The New Indian Journal of OBGYN. 2021; 8(1): 33-8. 
typical of the preoperative period, especially for the patient's first few surgical experiences. Studies have shown that high preoperative anxiety levels can lead to increased postoperative analgesic requirement, prolonged hospital stay, significant contribution to adverse peri-operative outcome and poor patient satisfaction.

The present study, therefore, explore the effects of hysterectomy on the quality of life in two different groups of women, one with and one without pre- operative education, and its effect on anxiety and depression both pre and post operatively.

\section{Methodology}

This was a hospital based study on patients admitted in gynecology ward in a rural hospital for elective hysterectomy. In this study preoperative education was done with the help of information booklet.

We formed two groups A and B each comprising of 48 patients each. The patients were randomly assorted into these groups, by asking them to pick up a number $(1=\mathrm{A} ; 2=\mathrm{B})$ which then decided their group. The women included in the study were in the age group of 38-70 years undergoing hysterectomy for benign conditions. Those included were willing to be part of study and could understand and comprehend. Those excluded from the study were undergoing surgery for malignant conditions, suffering from psychiatric disorder and suffering from chronic pain

Written informed consent was obtained from all participants who showed interest in the study. Participants were assured that their responses would be confidential and reminded that their participation in the interview was voluntary. Women of group A (intervened) were then educated by verbal counseling session, explaining them about the kind of surgery, procedure, how to prepare for the surgery, what to expect after the surgery, and the importance of the surgery. An information booklet displaying pictures of the same was shown to them. Their queries (if any) were answered in the language that they understood. Women of group B (control) were only given preoperative advice. Each woman was then asked to fill a questionnaire pre-operatively i.e. the day before surgery. The illiterate women were verbally conveyed about the information and their responses were taken by the interviewer.

A pre-validated and predesigned questionnaire was discussed with the women and their response was taken. It comprised of - general information, gynecological history, relationship status and pre-operative anxiety monitoring in accordance to HADS (Hospital Anxiety and Depression
Scale). The HADS is a fourteen item scale that generates: Seven of the items relate to anxiety and seven relate to depression. Scoring for each item ranges from zero to three, with three denoting highest anxiety or depression level. A total subscale score of $>8$ points out of a possible 21 denotes considerable symptoms of anxiety or depression. The HADS manual indicates that a score between 0 and 7 is "normal," between 8 and 10 "mild," between 11 and 14 "moderate," and between 15 and 21 "severe."

The responses of second questionnaire were taken from the women on fifth postoperative day. It consisted of - postoperative impact on psychological health, post-operative anxiety monitoring in accordance to HADS.

There was no difference of any kind in the type of questions for both the groups, women of both the group were asked questions in similar pattern with no variation or stressing upon certain points, therefore interviewer's bias was avoided. The pre-operative monitoring in women would focus on the basic knowledge about hysterectomy and social as well as family support for the operation.

Post-operatively we once again assessed the anxiety and depression levels in women by HADS. This would enhance our knowledge about the difference in level of anxiety post operatively in both the groups.

Statistical analysis: Data was analyzed using SPSS-16. A "P"-value less than 0.05 were considered significant. Two tailed $t$ test was used. Chi square test was used for dichotomous or categorical variables. Odds ratio was calculated in addition to confidence interval. Multivariate analysis of covariates was performed

\section{Results}

All together 96 women took part in the study. Mean age in the intervened group was 46 (std. error of 1.31) and in control groups was 47.5 (std. error $=1.44$ ). Mean education on the basis of class of schooling in the intervened group was 6.17 and in control group was 5.79 class of schooling. Mean age of menarche was 13.98 years in intervened group and 14.13 years in control group and mean age of menopause in the intervened group was 49.88 years, and in control group was 49.82 years. Total of only 19 women were postmenopausal. The above data is tabulated in table 1 . There is no variation in the baseline variables. $53.19 \%$ of the intervened women and $45.83 \%$ of control group were diagnosed with dysfunctional uterine bleeding (DUB). Other diagnosis includes fibroid - intervened (21.28\%) and control (29.17\%), and prolapsed uterus - intervened (17.02\%) and control (14.58\%). $81.25 \%$ of intervened women, and $89.58 \%$ 
Table 1: Mean values for demographic factors Demographic factors (total observations 96) Mean age in years

Mean age of menarche in years

Mean age of menopause ( total observations -19) in years

Mean education

Preoperative diagnosis

DUB

Fibroid

Adenomyosis

Prolapsed uterus

PID

Ovarian cyst

DUB - Dysfunctional uterine bleeding, PID - Pelvic inflammatory disease

of control group were unaware of the approach of the surgery and $93.75 \%$ women did not know whether there was associated oophorectomy performed.

Anxiety and depression: We had assessed anxiety and depression of the intervened and control groups both pre and post-operatively. The assessment was in accordance with HADS. Accordingly, mean of pre-operative anxiety in the intervened group was $6.81 \pm 2.69$ as compared to the control group $9.13 \pm 3.36$. This was statistically significant as assessed by two sample $t$ test, with $p$ value of 0.0003 . Mean of post-operative anxiety was $6.79 \pm 3.46$ in intervened group, and $9.71 \pm 3.16$ in control group, which is statistically significant; with $\mathrm{p}$ value of 0.0001 . Mean of pre-operative depression was $8.02 \pm 3.46$ in the intervened group and 9.93 \pm 4.55 in control group, statistically significant; with $\mathrm{p}$ value 0.022 . Lastly, mean of post-operative depression of $6.85 \pm$ 3.98 in intervention group, and $10.48 \pm 4.82$ in the control group, statistically significant; with $\mathrm{p}$ value of 0.0001 (table 2).

Table 2: Comparative analysis for HADS score

\begin{tabular}{llllll}
\hline Variables & $\begin{array}{l}\text { Intervened } \\
\text { group }\end{array}$ & $\begin{array}{l}\text { Control } \\
\text { group }\end{array}$ & t value & $\begin{array}{l}\text { P } \\
\text { value }\end{array}$ & $\begin{array}{l}\text { Degrees of } \\
\text { freedom }\end{array}$ \\
\cline { 2 - 3 } & $\begin{array}{l}\text { Mean value } \pm \\
\text { std. deviation }\end{array}$ & $\begin{array}{l}\text { Mean value } \pm \\
\text { std. deviation }\end{array}$ & & & \\
\hline Pre-operative anxiety & $6.81 \pm 2.69$ & $9.13 \pm 3.36$ & -3.55 & 0.0003 & 94 \\
Post-operative anxiety & $6.79 \pm 3.46$ & $9.71 \pm 3.16$ & -4.04 & 0.0001 & 94 \\
Pre-operative depression & $8.02 \pm 3.46$ & $9.93 \pm 4.55$ & -2.32 & 0.022 & 94 \\
Post-operative depression & $6.85 \pm 3.98$ & $10.48 \pm 4.82$ & -4.01 & 0.0001 & 94 \\
\hline
\end{tabular}

In the intervention group the women with normal, borderline and abnormal anxiety value pre-operatively were 28,16 and 4 respectively as compared with that of control group were 16, 14 and 18 respectively. This was statistically significant with fisher's exact of 0.002 . In the intervention group the women with normal, borderline and abnormal anxiety post-operatively were 24,19 and 5 respectively as compared with that of control group were 13, 16 and 19 respectively. This was statistically significant with fisher's exact of 0.003 (figure 1a, 1b). In the intervention group the women with normal, borderline and abnormal depression pre-operatively

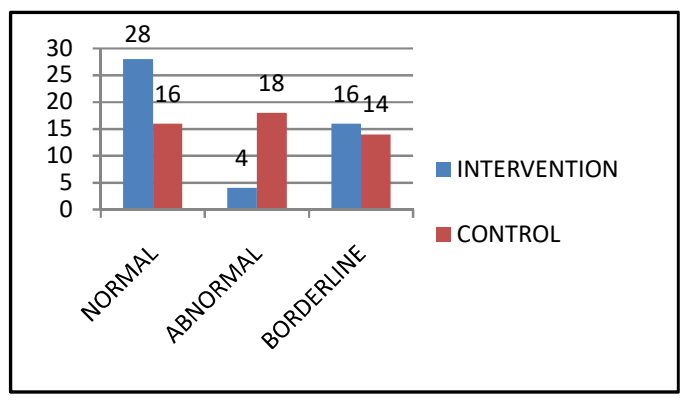

(a)

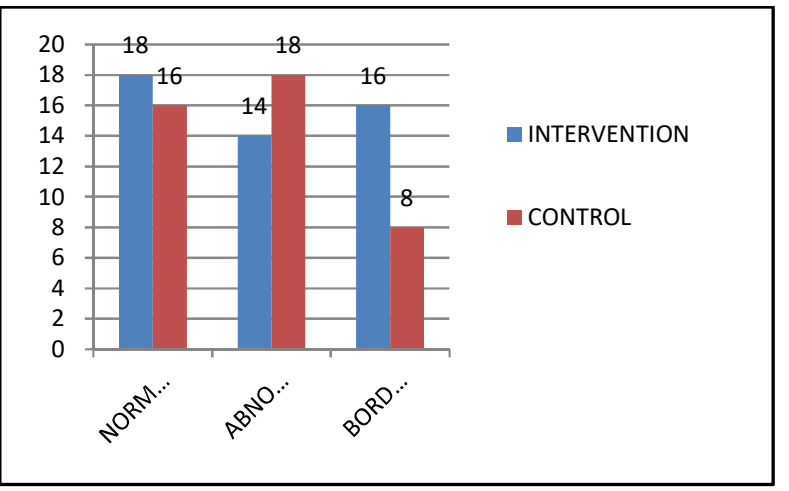

(b)

Figure 1: Comparing the HADS score in the intervention and control group (a - Pre -operative Anxiety; b - Post-operative anxiety) post-operatively were 25,13 and 10 respectively as compared with that of control group were 15, 8 and 25 respectively. This was statistically insignificant with fisher's exact of 0.006 .

Post-operative perception: Postoperation, 47 of the intervened women and all the women of control group were satisfied with the surgery, statistically insignificant; fisher's exact of 1. For 20 intervened patients and 6 patients of control group were very acceptable with the fact that the uterus was no longer present in the body post surgery, statistically significant; fisher's exact of 0.001 . 45 of the 
intervened group were not feeling depressed whereas 19 patients of the control group were feeling depressed, statistically significant; fisher's exact of 0.0005 . 46 of the intervened group were not feeling anxious whereas 24 women of the control group were feeling anxious, statistically significant; fisher's exact of 0.0005 . When they were asked about are they no longer feeling like women, all the patients in the intervened group did not have any such perception, whereas 10 women of control group felt that deprivation, statistically significant with fisher's exact of 0.001. There was statistical significance in the perception about loss of child bearing ability and sudden menopause (table 3).

\begin{tabular}{|c|c|c|c|c|c|c|}
\hline \multirow[t]{2}{*}{ Variables } & \multicolumn{2}{|c|}{$\begin{array}{l}\text { Intervention } \\
\mathrm{N}=\mathbf{4 8}\end{array}$} & \multicolumn{2}{|c|}{$\begin{array}{l}\text { Control } \\
\mathrm{N}=48\end{array}$} & \multirow[t]{2}{*}{$\begin{array}{l}\text { Fisher's } \\
\text { exact }\end{array}$} & \multirow{2}{*}{$\begin{array}{l}\text { 1-sided } \\
\text { Fisher' } \\
\text { exact }\end{array}$} \\
\hline & Yes & No & Yes & No & & \\
\hline Satisfaction & 47 & 1 & 48 & 0 & 1.000 & 0.500 \\
\hline Perception & 47 & 1 & 46 & 2 & 1.000 & 0.500 \\
\hline \multicolumn{7}{|l|}{ Psychological impact post-surgery } \\
\hline 1. Depression & 3 & 45 & 19 & 29 & 0.0005 & 0.0005 \\
\hline 2. Anxiety & 2 & 46 & 24 & 24 & 0.0005 & 0.0005 \\
\hline 3. No feeling of womanhood & 0 & 48 & 10 & 38 & 0.001 & 0.001 \\
\hline 4. Grief of child bearing ability & 1 & 47 & 2 & 46 & 0.605 & 0.470 \\
\hline 5. Grief of loss of menstrual cycle & 0 & 48 & 2 & 46 & 0.228 & 0.228 \\
\hline \multicolumn{7}{|l|}{ 6. Feeling about removed uterus } \\
\hline 1.Very acceptable & 20 & & 6 & & 0.001 & - \\
\hline 2.Acceptable & 28 & & 38 & & & \\
\hline 3. Not acceptable & 0 & & 4 & & & \\
\hline
\end{tabular}

There was no mortality or complication in any of the women, and the hysterectomy operation was not associated with any other surgical intervention.

\section{Discussion}

Present study reveals that there is a positive impact of pre-operative education on post-operative recovery, in women undergoing elective hysterectomy. The mean score in intervention group for pre-operative anxiety and postoperative anxiety in accordance with HADS was significantly less as compared to in the control group. In case of depression scores there was a significant rise in the score in the control group from pre-operatively to post-operatively and a significant fall was seen in that of intervention group from pre-operatively to post-operatively.

Women, being from rural areas of India were educated only till the primary level of schooling. This result is consistent with the other studies which stated that hysterectomy appears to be associated with low education, high parity, and a history of multiple miscarriages. The influence of these factors varies depending on the primary indication for the hysterectomy. Due to low level of education women may show readiness for the surgery $(100 \%)$. The possible explanation for these findings are that less educated women may delay health seeking behavior for casual gynecologic problems thereby keeping hysterectomy as last resort option of treatment or may be they are advised hysterectomy as the primary option for treatment by their physicians.

Another important finding from the study is indication for the surgery, wherein DUB followed by fibroid was the most common indication of hysterectomy, this is in odds with the findings in other studies where most common indication for abdominal approach of the surgery was fibroid uterus (total 39.9\% and out of abdominal 52.7\%), while for vaginal approach it was uterine prolapse (total $16.3 \%$ and out of vaginal $91.5 \%)^{5}$. A study in USA also found fibroid $(60 \%)$ to be the most common indication and the 2nd common indication was prolapse $(11 \%)^{6}$. The data from Hong Kong, fibroid was a higher proportion of indication for abdominal (73.7\%) and genital prolapse was most common indication $\quad(96.2 \%)$ for vaginal hysterectomy ${ }^{7}$. In Pakistan also the most common reason for hysterectomy was fibroid $(33 \%)$ and then was uterine prolapse (19\%) and DUB in $18(12 \%)^{8}$. In fibroids were the most common reason for performi hysterectomy (23\%); however there it was followed by DUB $(14.9 \%)^{9}$. In a study from Canada, however, the commonest indication of hysterectomy was DUB (26.4\%), followed by fibroid uterus $(16.0 \%)^{10}$.

A study carried out by Patrick Callaghan and Ho Cheung $\mathrm{Li}$ to test the effect of cognitive interventions with information given pre-operatively on post-operative outcomes in Chinese women undergoing elective hysterectomy. The study was carried out in Chinese women divided into two groups case (48) and control (48). The outcome measurement was in the form of post-operative anxiety (Chinese State-Trait Anxiety Inventory and mean arterial blood pressure), post-operative request for analgesia (drug record), post-operative pain (visual analogue scale), satisfaction (Chinese Patient Satisfaction Questionnaire); wherein the cases were given cognitive distraction and cognitive reappraisal with information and the control group was provided with information only. The results of this study demonstrate that the use of cognitive distraction and reappraisal may have significant clinical benefits. There were no significant differences between the control and 
experimental groups on baseline state anxiety scores, moreover among the study group $32 \%$ had completed lower secondary education and $31 \%$ had completed upper secondary education. There is no statistical evidence to support anxiety score ${ }^{11}$.

There is another study by Dr. Susan M Cohen; purpose of this study was to describe psychosocial adaptation as measured by anxiety, hostility, depression, self-esteem, body image, and sexual satisfaction in women following abdominal hysterectomy. The study reported that anxiety, depression, and hostility were highest in the immediate postoperative period and decreased over the eight weeks, majority of women in the study having high self-esteem scores. The scores remained stable over the study period ${ }^{12}$. But there was no intervention as to decrease the anxiety in patients both pre-operatively and post-operatively.

It is evident that anxiety scores in accordance with HADS were significantly less both pre-operatively and postoperatively in the intervened group as compared to the control group, the same was seen in case of depression scores. The results are consistent with previous studies. These findings support the view that, information plus cognitive interventions will decrease anxiety levels in the post-operative period ${ }^{13}$. Breemhaar argued that the education and support provided preoperatively to the women had positive effect on their physical and psychosocial well being before and after surgery because education and support maintain and increase the patient's feelings of control ${ }^{14}$. Another study was performed in China with an objective to determine whether a preoperative education intervention designed for Chinese cardiac patients can reduce anxiety and improve recovery. It was a randomised control trial and HADS was used for evaluation of anxiety and depression. The study stated that participants who received preoperative education experienced a greater decrease in anxiety score (mean difference -3.6 points, $95 \%$ confidence interval -4.62 to $-2.57 ; \mathrm{P}<0.001)$ and a greater decrease in depression score (mean difference -2.1 points, $95 \% \mathrm{CI}-3.19$ to -0.92 ; $\mathrm{P}<0.001)$ compared with those who did not. ${ }^{15}$ Whereas a randomised controlled trial in Korea was done to examine the effect of preoperative education using multimedia on the level of pain, uncertainty, anxiety and depression in hysterectomy patients. Experimental group showed lower level of post-operation pain in 1 hour $(\mathrm{t}=-5.08, \mathrm{p}<.001)$ and 24 hours $(\mathrm{t}=-5.20, \mathrm{p}<.001)$ but not 48 hours $(\mathrm{t}=-0.91$, $\mathrm{p}=.368)$. Uncertainty showed significant interaction effect of group by time $(\mathrm{F}=4.16, \mathrm{p}=.018)$. Thus the study concluded that preoperative education using multimedia for patients with hysterectomy would be effective in lowering patients' level of pain, uncertainty, anxiety and depression ${ }^{16}$. In another review, Shuldham found no demonstrable impact from preoperative education of the patient having coronary artery bypass surgery and there was further research suggested ${ }^{17}$. There was no significant impact of patient education on anxiety, pain or depression was found. However, the length of hospital stay was different significantly in the women who were given pre-surgical education ${ }^{18}$.

Moreover, the clinical relevance of these results is mainly related to the influence of anxiety on surgical stress. Indeed, anxiety is a psychological aspect of surgical stress. This latter can have many physical consequences such as increased sympatho-adrenal medullary activity and impaired immune function with increased susceptibility to disease. The stress response is multisystemic and involves the activation of neuroendocrinologic and metabolic system ${ }^{19}$. The extent of the perioperative stress response is directly correlated to the magnitude of surgery. Therefore in the present study it is evident that pre-education reduced the anxiety and depression in patients both pre-operatively, and post-operatively, thereby disapproving the use of anxiolytics. Significant low levels of anxiety and depression have impact on post-operative pain perception.

\section{Conclusion}

Present study reveals that there is a positive impact of pre-operative education on post-operative recovery, in women undergoing elective hysterectomy. This study concludes that by providing women with basic information about the surgery there is significantly less anxiety and depression experienced by the women both pre-operatively and post-operatively.

\section{Conflict of interest: None. Disclaimer: Nil.}

\section{References}

1. Wu JM, Wechter ME, Geller EJ, Nguyen T V, Visco AG. Hysterectomy Rates in the United States, 2003. Obstet Gynecol. 2007;110(5): 1091-5.

2. Singh A, Arora AK. Why Hysterectomy Rate are Lower in India. Indian J Community Med. 2008 Jul; 33(3): 196-7.

3. Wong LP, Arumugam K. Physical, psychological and sexual effects in multi-ethnic Malaysian women who 
The New Indian Journal of OBGYN. 2021 (July-December);8(1)

have undergone hysterectomy. J Obstet Gynaecol Res. 2012 Aug 1; 38(8):1095-105.

4. Zigmond AS, Snaith RP. The hospital anxiety and depression scale. Acta Psychiatr Scand. 1983; 67: 361-70.

5. Pandey D, Sehgal K, Saxena A, Hebbar S, Nambiar J, Bhat RG. An Audit of Indications, Complications, and Justification of Hysterectomies at a Teaching Hospital in India. International Journal of Reproductive Medicine. 2014; 2014:279273. Available from: https://doi.org/ $10.1155 / 2014 / 279273$

6. Broder MS, Kanouse DE, Mittman BS, Bernstein SJ. The appropriateness of recommendations for hysterectomy. Obstet Gynecol. 2000; 95(2):199-205.

7. Leung PL, Tsang SW, Yuen PM. An audit on hysterectomy for benign diseases in public hospitals in Hong Kong. Hong Kong Med J. 2007;13(3):187.

8. Toma A, Hopman WM, Gorwill RH. Hysterectomy at a Canadian tertiary care facility: results of a one year retrospective review. BMC Womens Health. 2004;4(1): 10 .

9. El-Toukhy TA, Hefni MA, Davies AE, Mahadevan S. The effect of different types of hysterectomy on urinary and sexual functions: a prospective study. J Obstet Gynaecol (Lahore). 2004;24(4):420-5.

10. Williams RD, Clark AJ. A qualitative study of women's hysterectomy experience. J Womens Health Gend Based Med. 2000; 9(2, Supplement 2):15-25.

11. Callaghan $\mathrm{P}, \mathrm{Li} \mathrm{HC}$. The effect of pre-operative psychological interventions on post-operative outcomes in Chinese women having an elective hysterectomy. Br J Health Psychol. 2002;7(2):247-52.

12. Cohen SM, Hollingsworth AO, Rubin M, Graff BM, Thomas JS, Welz HK, Wehry LE. Psychosocial Adaptation During Recovery from Hysterectomy.
Webmed Central Obstetrics And Gynaecology. 2011;2(3):1-8.

13. Young R, DE Guzman CP, Matis MS, McClure K. Effect of preadmission brochures on surgical patients' behavioral outcomes. AORN J. 1994; 60(2): 232-41.

14. Breemhaar B, Van den Borne HW. Effects of education and support for surgical patients: the role of perceived control. Patient Educ Couns. 1991;18(3):199-210.

15. Guo P, East L, Arthur A. A preoperative education intervention to reduce anxiety and improve recovery among Chinese cardiac patients: a randomized controlled trial. Int J Nurs Stud. 2012; 49(2):129-37.

16. Jeon S, Park H. Effect of preoperative education using multimedia on pain, uncertainty, anxiety and depression in hysterectomy patients. Korean J Women Heal Nurs. 2016; 22(1): 39-47.

17. Shuldham CM. Pre-operative education for the patient having coronary artery bypass surgery. Patient Educ Couns. 2001; 43(2):129-37.

18. Shuldham CM, Fleming S, Goodman H. The impact of pre-operative education on recovery following coronary artery bypass surgery. A randomized controlled clinical trial. Eur Heart J. 2002;23(8): 666-74.

19. Godoy LD, Rossignoli MT, Delfino-Pereira P, GarciaCairasco N, de Lima Umeoka EH. A Comprehensive Overview on Stress Neurobiology: Basic Concepts and Clinical Implications. Front Behav Neurosci. 2018;12:127.

\author{
Shuchi Jain ${ }^{1}$, Shruti Pansare ${ }^{2}$ \\ ${ }^{1}$ Professor of Obstetrics \& Gynaecology, Mahatama \\ Gandhi Institute of Medical Sciences, Sevagram, \\ Wardha, Maharashtra, India; ${ }^{2}$ Intern, Mahatama \\ Gandhi Institute of Medical Sciences, Sevagram, \\ Wardha, Maharashtra, India.
}

